\title{
Simulation of Dynamic Property for Wave Reflection in Basin over a Half Infinite Space by Staggered Grid RFFT Differentiation
}

\author{
Zhixin Zhao, Jiren Xu \\ Institute of Geology, Chinese Academy of Geological Sciences, Beijing, China \\ Email: Zhaozx1010@hotmail.com
}

Received 7 April 2015; accepted 16 May 2015; published 20 May 2015

Copyright (C) 2015 by authors and Scientific Research Publishing Inc.

This work is licensed under the Creative Commons Attribution International License (CC BY). http://creativecommons.org/licenses/by/4.0/

(c) (i) Open Access

\begin{abstract}
Simulations for seismic wave propagation in the sedimentary basin over half-infinite rock space were performed in this analysis. The results indicated that the basin shape in heterogeneous structures affected wave dynamic characteristics in propagating. The refraction wave from the rock brought the multireflection wave and the secondary surface wave in the basin. The waves oscillating up-down within the basin shook the buildings time after time resulting in heavy damage. The geometrical focusing by basin corners and the physical interference between waves significantly amplified the ground motions. The location of peak ground motion due to the interference was attributable to the apparent velocity difference between the secondary surface wave and the body wave. The late-arrival waves also resulted in the peak ground motion. The frequencies of the late-arrival waves of multireflection might cause dispersion. The late-arrival conversion waves with various frequencies widened the frequency band of seismic input waves shaking buildings and could seriously damage buildings with corresponding intrinsic frequencies.
\end{abstract}

\section{Keywords}

Late-Arrival Wave, Multireflection, Apparent Velocities, Frequency Band Widen, Peak Ground Motion, Damaging Buildings

\section{Introduction}

Numerical simulation of wave propagation in a heterogeneous medium has been employed widely in various geophysical research projects [1]-[7]. Many results of the strong ground motions have been reported in order to mitigate earthquake damages [8]. The wave propagation in the heterogeneous structure formed by the sediment, 
or alluvial layer, lying upon the rock layer is an interesting topic for seismological and aseismic studies [9] [10] because the sedimentary basins of complex structures are distributed widely over the continent. Moreover, it is worth mentioning that many urban districts are located in or near such basins. Earthquake damage related to basin became a serious problem for modern cities as industry develops and the pace of urbanization speeds up [11] [12]. The bottom shapes of basins are usually quite different from each other. Many basins are beside mountains or foothills. Some of them are even typically bounded by active fault zones in the tectonic sense. The different structure of a basin can affect the earthquake damage scale and its distribution. Severe earthquake damage can happen in basin regions far away from an earthquake fault, even if low seismicity exists in the basin [13] [14]. Therefore, many numerical simulation studies on ground motion around special basins have been carried out [15]-[19]. The earthquake hazard and strong ground motion around heterogeneously sedimentary basins have been extensively discussed. Some results suggested that earthquake damage and complicated intensity distributions might be related to the geological structure [20] [21]. Some recent results suggested that the secondary surface wave could be generated during wave propagation in an unhomogeneous basin. The secondary wave also affected the strong ground motion. The simulations of secondary surface waves and their spatial interference with the body wave were introduced to ground motion study in the alluvial fan structure [22]. The problem about reflection wave is also an important topic as one investigates the relation between the strong ground motion and the secondary waves in the basin structure. The propagation apparent velocity of a reflection wave might be different from that of the incidence wave at some time [23]. The reflection wave shocks to the buildings on the surface and extended the duration of the shocks too. The investigation of secondary reflection waves is of significance for analyses of the strong ground motion in a heterogeneous medium [24].

Unlike previous studies focused on lateral variations of strong ground motion we are going to investigate dynamic characteristics of the waveforms besides spatial variations of waveform amplitudes in detail. The reason is that earthquake damage is closely related to the spectra of input waves, duration and effect on buildings too. The seismic wave propagation processing in the inner basin and the effects on ground motion by the incidence and late-arrival conversion waves are discussed fully in the present analysis. The structure formed by the sedimentary medium over the rock layer is adopted for the simulation of strong ground motion in this analysis. We prefer a cross section transecting the sedimentary basin to demonstrate wave propagation simulations. The wave characteristics in the propagation process were investigated by employing snapshots, waveform gather and ground motion distribution over the basin structure in the following sections.

\section{Simulation Technique}

The wave simulations involved the recurrence and conversion wave phases in wave propagation. The wave propagation and its ground motions in a heterogeneous structure were calculated as a 2D-SH wave problem using the pseudo-spectral method with a staggered grid RFFT differentiation operator (SGRFFTD) [22] [25]-[27]. This computerized modeling method has been widely employed in geophysical simulation because of its efficiencies in memory cost [28]. The predominant period in source function may be selected according to the intrinsic period of the studied object. The size of the grid should be fitted to the resolution of study. Time step length $\Delta t$ can be determined based on the formula $v \Delta t / \Delta x<\sqrt{2 / \pi}$. The maximum frequency of the simulation technique calculated by $f_{\max }=V_{\min / 2 \Delta x}$ should not be less than that of the study objective. A simplified line source employed in the simulation may be beneficial for investigating the relationship between the wave propagation and the geological structure, although the wave field variations are also related to many seismic parameters [29]. The line source force perpendicular to the profile in Figure 1 is taken as a Gaussian-shaped spatial function with a Ricker wavelet time source function having a predominant frequency of $3.5 \mathrm{~Hz}$. In the discrete numerical simulation, the model in the present analysis was sampled using $256 \times 128$ grids at $100 \mathrm{~m}$ horizontal (x) and depth (z) spacing. According to velocity structure and grid spacing, the limiting frequency in the simulation is about $6.0 \mathrm{~Hz}$, and the time interval is taken as 0.03 seconds. Seismic waveforms with a duration of 18 seconds were simulated. The absorbing boundary condition with twenty grids was applied to the lateral and bottom edges of the spatial grid [30]. The exponential absorption width was twenty grids in the above edges. The free-surface condition was approximated by including 128 zero shear wave velocity points above the upper surface of the model in vertical derivatives of stress. The symmetric differentiation method was applied for vertical differentiation of displacement to stabilize the derivatives in the surface nodes. 


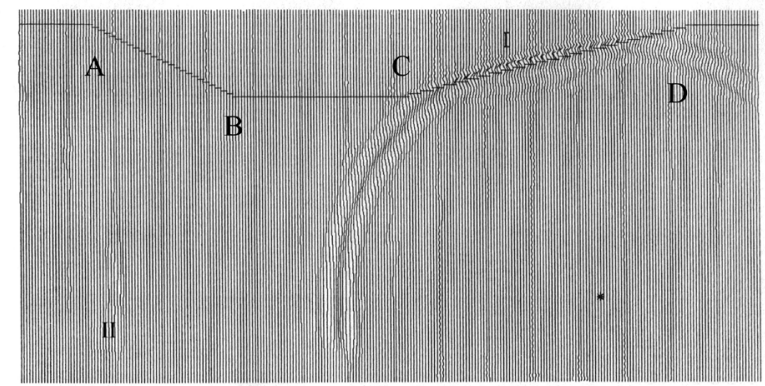

$\mathrm{t}=2.7 \mathrm{~s}$

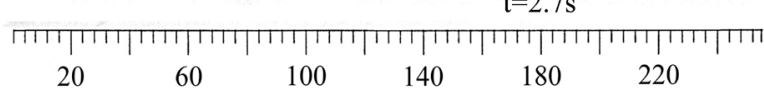

Figure 1. Model structure formed by the sediment basin over the half infinite rock space and the seismic acceleration wave propagation snapshots at 2.7 seconds. Broken line ABCD: boundary between the granite rock (II) and sediment soil (I) layers. Asterisk $\left(^{*}\right)$ : shake source. The number under the abscissa: Observation site No.

\section{Propagation of Multi-Reflection Waves}

Some late conversion waves related to the ground motion occur during the propagation in the basin. In order to clarify the causative mechanisms of the late-arrival conversion waves concerning the basin structure it is necessary to investigate the wave propagation process. Figure 1 depicts the basin structure scheme for the sediment basin layer over the half infinite rock space with the simulated snapshot of the seismic acceleration wave propagation starting just from the source. Broken line ABCD represents the boundary between the sediment soil (I) and granite rock (II) layers, viz. the bottom of the basin. The asterisk in the lower right part of region II represents the source location. The seismic wave velocities and densities of the sedimentary basin and rock are shown in Table 1. In the snapshot of Figure 1 the wave propagated radiantly as a spherical wave front in the rock region II at 2.7 seconds. It could also be seen that the spherical wave was refracting into the sedimentary layer (region I) at the segment CD of the basin bottom boundary at that time. The original spherical wave front became flatter, converting seemingly a plane wave front partially at the segment CD. The conversion wave is named as the refraction wave S1 hereafter. Simultaneously a weak wave with similar spherical wave front reflected back the rock layer II under segment CD of the boundary.

Figure 2 shows other simulated snapshots of the seismic acceleration after $2.7 \mathrm{~s}$. In the snapshot at 5.4 seconds in Figure 2(a) it is clearly seen that the original spherical wave in rock region II almost approached corner A on the left side of the basin bottom. The corresponding reflection wave back layer II can be seen under site B. The refraction acceleration wave S1 upgoing at CD region in Figure 1 converted already the leftgoing wave and was propagating leftward over segment BC in the sedimentary region I at $5.4 \mathrm{~s}$. It looked like a plane wave front there. The lower end of the front of wave S1 seemed to fold upward above segment $\mathrm{AB}$ as the wave S1 passed through the corner B in Figure 2(a). The amplitude of wave S1 over segment AB was likely to be less than that over segment BC. This might be due to the refraction angle of wave S1 from layer II to layer I at segment $A B$ was different from those at segments $B C$ and $C D$. At the upper end of wave $S 1$ in Figure 2(a), the leftgoing wave S1 reflected back region I at the air free surface over corner C, forming a downgoing wave propagating leftward following S1, which was taken as the downgoing reflection wave of S1 at the surface. When the reflection wave of S1 traveled to segment CD of the bottom in Figure 2(a) it reflected strongly upward. This upward wave was taken as multi-reflection seismic acceleration wave S2. Simultaneously, however, it looked as if hardly any wave refracted into hard rock layer II from the downgoing reflection wave of S1 in layer I. Only a very weak refraction wave was transmitted to the rock layer at the site generating wave S2 through segment CD, compared with the amplitude of reflection wave S2.

Similarly the strong multi-reflection seismic acceleration wave S3 following wave S2 occurred in Figure 2(b) and Figure 2(c). The downgoing reflection waves at the free surface of S2 and S3 can also be seen in Figure 2(b) and Figure 2(c). In the snapshot 8.1s in Figure 2(b), the leftgoing wave S1 in Figure 2(a) has already disappeared. The remaining downgoing surface reflection wave of S1 links to the lower end of wave front S2. The 
(a)

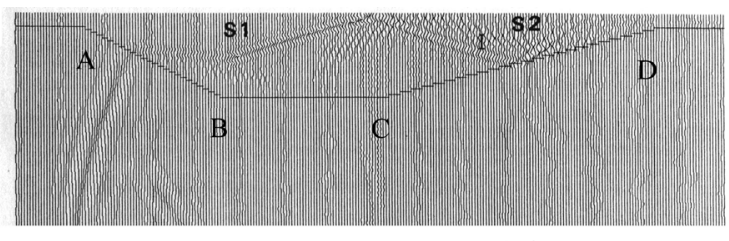

$\mathrm{t}=5.4 \mathrm{~s}$

(b)

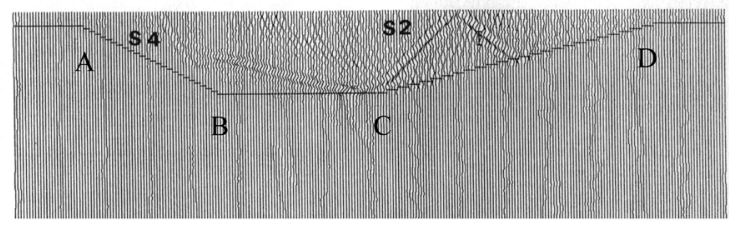

$\mathrm{t}=8.1 \mathrm{~s}$

(c)

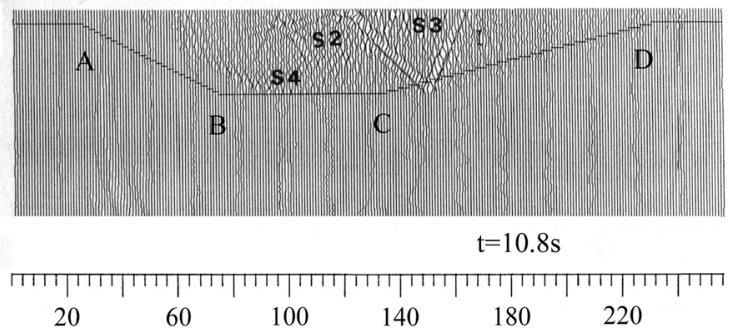

Figure 2. Simulated snapshots at 5.4 (a), 8.1 (b) and 10.8 (c) seconds for seismic acceleration wave propagations. S1, S2, S3, and S4 are seismic phases. Other symbols have same meanings as those in Figure 1. The number under the abscissa: Observation point No.

lower end of wave front S2 folded up when it passed the corner C. The different slopes of the wave front represented the apparent velocity variation (the velocity of wave propagating along the surface). This was because the reflection plane in the bottom generating wave S2 varied from segment CD to BC. It could be seen that a multi-reflection wave formed by waves S2 and S3, etc., traveled leftward in t he basin evidently. The wave S3 was developed well in shot 10.8s. Simultaneously with the generating of multireflection wave S3, however, it looked as if hardly any wave refracted into hard rock layer II from the downgoing reflection wave of S2 in Figure 3(b) and Figure 3(c), similar to the case with wave S2 generation in Figure 2(a). Some very weak refraction wave perhaps transmitted into the rock at the generation site of S3 through the basin bottom in Figure 2(b) and Figure 2(c).

It can also be seen that the leftgoing wave S3 reflected back the sediment layer at the surface in shot 10.8s. The downgoing reflection wave of S3 propagated following S3. The left-going waves S1, S2 and S3 passed through the surface one by one, with their own apparent velocities. Such sequential oscillation shook the constructions on the surface multiple times, and probably could result in heavy damage.

In Figure 2(b), clear seismic acceleration wave S4 appeared over segment AB and propagated rightward, crossing the downgoing reflection wave of S1. Wave S4 was the secondary surface wave which was converted at the basin edge after the spherical wave from the granite rock layer refracted upward into the shallow sedimentary layer I left of corner A. The secondary surface wave S4 was propagated in the opposite direction to that of S1 as mentioned above. More conversion wave phases occurred and propagated along different directions in the basin in shot $10.8 \mathrm{~s}$ in Figure 2(c). If the rightgoing seismic wave S4 met the leftgoing wave S2 the interference between the secondary surface wave S4 and the reflection body wave S2 probably occurred at that location of the basin. This might have resulted in peak ground motion and heavy damage also. The locations of peak ground motions are perhaps attributable to the apparent velocity difference between the secondary surface wave and the body wave.

\section{Results and Discussion}

Figure 3(b) shows the simulated acceleration seismogram gather lasting 18 seconds at the surface of the model 


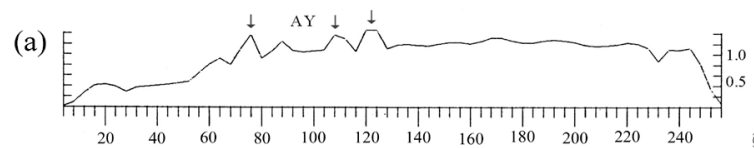

(b)

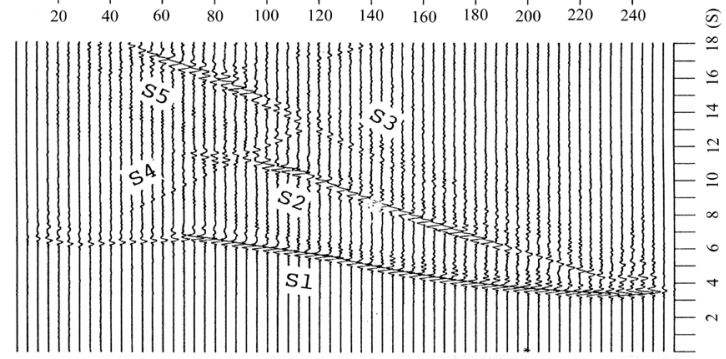

Figure 3. Simulated seismic acceleration waveform gather at the surface of the model in Figure 1(b), and the maximum amplitude distribution of the waveforms at the corresponding sites in panel (b) during the 18-second simulation period (a). The number under the abscissa: Observation point (grid). The number by the ordinate: simulation time (unit: second). ${ }^{*}$ in the lower right: the source. $\downarrow$ : peak amplitude. S1, S2, S3, and S4: same seismic phases in Figure 2. Notice: the maximum amplitudes in (a) and waveforms in (b) about 20 grids in the lateral and bottom edges of the model are no meaning because of the absorbing boundary condition with twenty grids.

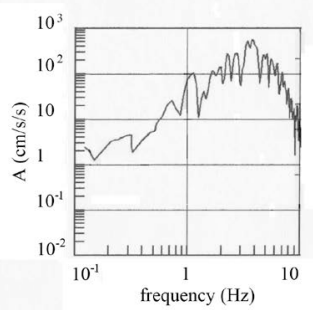

(a)

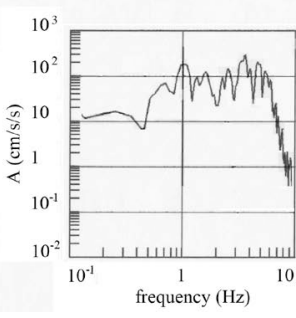

(b)

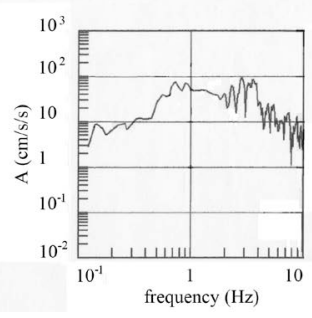

(c)

Figure 4. Spectrum analyses of the waveform during the period of great wave amplitude. (a): for waveform S1 between the 2nd and 6th second at the 120th observed site in Figure 3(b), (b): for waveform S2 between the 6th and 10th second at the 120th observed site in Figure 3(b), (c): for waveform S3 between the 13th and 17th seconds at the 96th observed site, respectively. The extremum amplitudes of waveform S1, S2 and S3 could be seen around 4.0 $\mathrm{Hz}, 2.9 \mathrm{~Hz}$, and $1.8 \mathrm{~Hz}$, respectively.

in Figure 1 (the 216 effectively simulated receiver points). Figure 3(a) gives the maximum amplitudes of waveforms observed at the corresponding sites in Figure 3(b). The seismic wave phase S1, S2 and S3 etc. mentioned above in Figure 2 are clearly seen in Figure 3 too. The ratio for the distance between two sites along the surface to their corresponding travel time difference indicates the wave's apparent velocity. The times intervals for waves to shake buildings on the surface were preferably related to the apparent velocity as mentioned above. This can also be seen directly in the travel time curve as shown in the seismic acceleration waveform gather in Figure 3. The travel time curves of converted wave S1, S2, etc., varied evidently from one another.

The travel time curve of the wave series S1 looks as though there are flat arcs in the right end in Figure 3(b) when wave S1 arrived just in the surface after about 3 seconds. There were great apparent velocities of S1 over there. The travel time curve gradually became precipitous along with the S1 leftgoing propagation. The apparent velocities became small from the 128th to 72th grids, about corresponding to the BC segment of the basin bottom in Figure 2. It became great left of the 76th grid again. The changes of the apparent velocity for waves S1 evidently were related to the corners D, C, B and A of the basin bottom boundary, similarly to the changes displayed by wave fronts in Figure 2. 
The travel time curve of wave series S2 did not vary as complicatedly as S1 did. The apparent velocity of S2 is less than that of S1 wavelet on a whole. The variation looked like uniformity right of the 100th grid. The apparent velocity of wave series S2 were likely great left of the observation point 100 . The apparent velocity of wave series S3 seems to vary much uniformly. The apparent velocity of S3 was less than those of wavelet S1 and S2 in Figure 3(b) usually. Apparent velocity of wave phases turned less and less in the medium together with reflection time increase in this analysis. The apparent velocity variations of waves were attributable to their reflection and refraction angles as the wave phases generated or propagated. The apparent velocity of rightgoing wave S4 probably was less than that of wave S3. For example, the average apparent velocity of the surface wave S4 between the 80th and 108th grid, the 12.5 grids/s was less than that of S3 by 2.1 grids/s.

It could roughly be seen that the amplitudes of waveforms revealed heterogeneous spatial distribution in Figure 1 and Figure 2. The temporal and spatial amplitude variations of the waveforms at the surface are clearly seen in Figure 3. With regard to the leftgoing wave S1, the amplitudes became suddenly little as the wave passed through observation point 76 over corner B (in Figure 2) of the basin bottom before 7 seconds in Figure 3(b). This may be because of the change of incidence angle of wave S1 that the spherical wave refracted in the basin from rock medium II passing through the corner B as shown in Figure 2. As for the amplitudes of wave phase S2, they were less than those of S1 on the whole. The waveforms of S2 were weak in the sites right of about the 190th grid before about 6 seconds, compared to those left of the 190th grid. The waveform of S2 almost vanished in the region left of the 64th grid.

The seismic acceleration waveforms of S3 were little right of the 112th grid in Figure 3(b). The amplitudes of leftgoing wave S3, however, became great about left of the 112th grid as it propagated through the corner B after the 12 seconds. Such amplitude change was attributable to the change of reflection plane of the S3 wave series propagating in the basin. The severe shaking of wave S3 after 12 seconds might result in heavier damage again following the strong shakes due to waves S1 and S2. The above results of temporal and spatial heterogeneous distribution for seismic ground motions are useful for the assessment of earthquake hazards and seismic risk.

In order to analyze spatial characteristics of strong ground motion further at the surface Figure 3(a) shows the maximum amplitudes of waveforms simulated at the corresponding sites in Figure 3(b). The maximum amplitude curve of waveforms fluctuated greatly with grids at the surface. The three peak amplitudes occurring in the observation grids 76, 108 and 124 marked by the downward arrows in Figure 3(a) respectively. The peak amplitude 1.42 at observation point 124 upper left of corner C in Figure 2 was attributable to waveform S1 around $5.8 \mathrm{~s}$ based on analyzing the waveforms in Figure 3(b). The peak amplitude 1.39 at grid 76 upper right of corner B (in Figure 2) simulated resulted from waveform S1 around $6.8 \mathrm{~s}$. Therefore, the above extrema of the amplitudes of S1 are probably attributable to the basin corner structure effects, e.g., corner focusing actions, resulting in heavy earthquake damage at the two sites. The grid difference between extremum point 124 and corner $\mathrm{C}$ is evidently different from that between extremum point 76 and corner B. The spatial location relationship between the corner of the basin bottom and the site of the extremum amplitude on the surface is attributable to the angle of the bend basin bottom corner in Figure 2 and wave incident angle. The third peak 1.38 at observation point 108 might be attributable to late arrival wave S2 around $10.9 \mathrm{~s}$ and/or perhaps generated by the interference between S2 and the secondary surface wave S4 as motioned above. This implies that the secondary wave might have resulted in peak ground motion and heavy damage again after wave S1 disappeared.

The three peak values were shown in Table 1 with their locations. It is noted that the peak values of the strong ground motion does not occur in the left region near the source in Figure 3, but in the right side away from the source. It implies that the geological structure in the heterogeneous medium more strongly affects the ground motion than the distance did in the present analysis.

The study on seismic wave deals with dynamic resolution. It is necessary to investigate the wave frequency because spectrum analysis is significant for aseismic research and mitigating damage. The destruction degree of buildings due to an earthquake is closely related to the frequency of input wave and some dynamic parameters. As mentioned above, the body wave refracted into the basin from the rock layer brought the multi reflection waves formed by wave S1, S2 and S3. The multi-reflection wave propagating in the soil basin structure $\left(\mathrm{V}_{\mathrm{s} 1}\right.$ in Table 2) over the half infinite rock space $\left(\mathrm{V}_{\mathrm{s} 2}\right.$ in Table 2) in Figure 2 is similar to the case of supercritical reflection of the body wave $\left(\mathrm{V}_{\mathrm{s} 1}<\mathrm{V}_{\mathrm{s} 2}\right)$ within the surface layer. It probably cause in the surface wave. The wave front of wave S1 looked narrow in the snapshot in Figure 2(a), being narrower than that of wave S2. The wave front widths of S1, S2 and S3 were also different from one another. Figure 3(b) depicts the simulated waveform 
Table 1. Wave velocity and density in each layer in Figure 1.

\begin{tabular}{ccccc}
\hline Layer & Vp $(\mathrm{km} / \mathrm{s})$ & Vs $(\mathrm{km} / \mathrm{s})$ & Density $\left(\mathrm{g} / \mathrm{cm}^{3}\right)$ & Reference \\
\hline I & 2.1 & 1.2 & 2.0 & Sediment \\
II & 6.0 & 3.5 & 2.6 & Granite \\
\hline
\end{tabular}

Table 2. Peak amplitudes of waveforms at the surface in Figure 3.

\begin{tabular}{ccccc}
\hline Peak No & Occurring grid & Time (s) & Maximum & Cause \\
\hline 1 & $120-124$ & 5.8 & 1.42 & S1 \\
2 & 76 & 6.8 & 1.39 & S1 \\
3 & 108 & 10.9 & 1.38 & S2, S4 \\
\hline
\end{tabular}

lasting 18 seconds gather at the surface observation points. Such frequency variations can be further identified in the waveform gather in Figure 3(b). The wave period changed even in the same wavelet, e.g., the wave periods of wave S2 left of the 190th grid were greater than those right of the 190th grid. The periods changed also in waves S1 and S3. The frequencies of the S2 wave series in the middle region between the 90th grid and the 190th grid are lower than those of the S1 series in the same region on average in Figure 3(b). Obviously, the frequencies of the multireflection wave series S3 with great amplitude between the 60th and 110th grid look lower than those of waveforms S2 and S1 simulated in the same grids as a whole. The late arriving wave S3 likely has the maximum period.

As example, we analyzed spectral characteristics of the main waveforms around their maximum periods in Figure 3(b), viz. analyzed spectral characteristics of the waveform S1 between the 2nd and 6th second and waveform S2 between the 6th and 10th second at the 120th observed site (seeing Figure 3) and waveform S3 between the 13th and 17th seconds at the 96th site. The spectrum analysis results of waveform S1, S2 and S3 in the periods and sites mentioned above were shown in left (a), middle (b) and right panels (c) in Figure 4, respectively. The great amplitudes of waveform S1, S2 and S3 could still be found about $4.0 \mathrm{~Hz}, 2.9 \mathrm{~Hz}$, and 1.8 $\mathrm{Hz}$, respectively. The corresponding periods for these frequencies approached to $0.25 \mathrm{~s}, 0.35 \mathrm{~s}$ and $0.56 \mathrm{~s}$. The finds imply that the frequency domains of great wave amplitude among the wave phases in Figure 4 get lower and lower according to the sequence of the wave phase arriving.

The apparent velocity and frequency of waves, such dynamic characteristics of waves varied in the propagation as mentioned above. The frequencies of the late arriving waveform S3 seem to get low. This may be a useful way to identify the reflection and multireflection waves in the medium layer. The finding shows that the complex basin structure can change not only the spatial distribution of the seismic wave field strength, but also deeply effect the period, etc, dynamic characteristics of waves in propagating. The late arrival conversion waves with various frequencies widen the frequency band of waves to shake buildings on the surface.

Seismic wave simulations in the model with the soil basin overlaying the half infinite rock layer space were performed in the present analysis. The potential effects upon the wave propagation and strong ground motion due to the regional structure characteristics of the basin were clarified in detail. The results showed that the spherical incident wave from granite rock refracting into the soil medium could not only excite a series of multireflection waves with the same propagation direction to the incident wave but also the secondary surface wave with the converse direction. The late-arrival seismic waves phases are probably attributable to the geometrical shape and geological structure.

Analyzing wave conversion status further, it can be found that the major energy from the source in the rock layer with high seismic velocity easily transferred into the soil layer with low velocity, and energy little reflected back to the rock layer as shown in Figure 1 and Figure 2. Whereas the wave was hardly refracted into the rock layer and reflected strongly back to the soil layer as the wave from the soil basin traveled to the bottom boundary of the basin. Such properties of energy transfer between media with high and low velocities are not only very significant for aseismic research but also for crustal velocity investigations and resource explorations [31] [32]. The energy of seismic waves barely returned to the rock layer but was consumed almost entirely in the sedimentary basin. The multireflection waves oscillated up and down within the basin as shown in Figure 2. The 
oscillations shook buildings over the basin structure time after time. This could make the earthquake damage severely.

The analysis of extrema of waveform amplitudes benefited assessments of earthquake hazard and seismic risk in urban areas. The amplitudes of all waves were distributed heterogeneously whether refraction wave or multireflection waves. Some peak amplitudes of waveforms at the surface due to the converted refraction wave S1 imply that geometrical focusing caused by corners in the basin bottom may significantly amplify ground motions. The interference between the reflection wave S2 and the secondary surface wave S4 coming from opposite directions further amplified the ground motions also. The late arrival wave also resulted in peak ground motion on the surface, as well as the initial refraction wave S1 in Figure 3. This is valuable information concerning ground motion for disaster prevention and aseismic research. The locations of interference peak ground motions are probably attributable to the apparent velocity difference rather than the velocity difference between the secondary surface and the body waves. The severe shaking of late arrival wave S3 might result in heavier damage again after the strong shakes due to waves $\mathrm{S} 1$ and $\mathrm{S} 2$.

The simulation showed that the geometry of the basin in the heterogeneous velocity structure not only changed the spatial distribution of the seismic wave fields, it also deeply affected the apparent velocity and wave frequency, such dynamic characteristics of the waves. The later the wave phase happened, the lower the apparent velocity was. The time interval for the waves S1, S2 and S3 shaking the buildings and the wave interference location are related to the apparent velocity. The apparent velocities varied with the bottom shape displaying the incidence and reflection angles of waves. Specially, spectrum analyses of the input waves S1, S2 and S3 were also significant for aseismic research and mitigating earthquake damage [33] [34]. The wave periods probably varied with propagation in the sediment layer based on the results in Figure 3 and Figure 4. The periods of waves S1, S2, S3 and S4 were different from one another. The frequency changed with location and time even for same wave phase. The highest frequency of wave S2 was lower than the highest one of wave S1. Similarly, the highest frequency of wave S3 was lower than the highest one of wave S2. The spectrum analysis also shows that the frequency domain of great wave amplitude for different wave phase gets lower and lower according to the sequence of the wave phase arriving.

The wave frequency dispersion was usually encountered in the wave propagation problem in the water layer over the half infinite space [35]. Such multi reflection wave S1, S2 and S3 propagating in sedimentary structure over the half infinite rock space, was similar to the case that the body wave supercritically reflected within the interface $\left(\mathrm{V}_{\mathrm{s} 1}<\mathrm{V}_{\mathrm{s} 2}\right.$ in Table 2). It probably generated the surface wave [36]. An incident wave from rock brought the multireflection waves with different frequencies in the sedimentary layer. It might be taken as frequency dispersion phenomenon. The late arrival conversion waves with various frequencies widened the frequency band of waves, resulting in heavy earthquake damage to more buildings with corresponding intrinsic frequencies. The spectral analysis for the waves may not only be significant for studies of strong ground motion but also for the elimination of the multireflection wave in resource exploration engineering.

The above properties about multireflection wave propagation, corner's focusing and wave interference in the basin can be seen in other cases [17] [22]. Figure 5 gave another result about wave propagation in basin to show relationship between propagation wave field and the parameters of basin structure. Figure 5(c) show the study results on soil amplification ratios for different half basin structures with 3 layers (a) and 4 layers (b) under the plane wave incidence case. The peak value accelerations of ground motion for (a) and (b) cases occurred in the different sites. The peak amplitude of (a) case was less than that of (b) cases here. The soil amplification ratio due to parameters of basin structure influences nonlinearly the seismic ground motion basically [37]. The further quantitatively scientific relationship might be expectable for research and engineering.

\section{Conclusions}

Simulation results for seismic wave propagation in the present analysis indicated that the heterogeneous velocity structures of the basin over the half infinite rock layer deeply affected the temporal-spatial distribution of dynamic characteristics of seismic waves besides the amplitudes.

The body wave from the rock layer refracted into the basin brought the multireflection waves formed by wave S1, S2 and S3, and the secondary surface wave S4. The seismic field in the basin revealed the newly complicated characteristics due to the occurrences of the multi-waves. The strong ground motions on the basin were closely related to multireflection waves and the secondary surface wave. As the results in the present analysis 


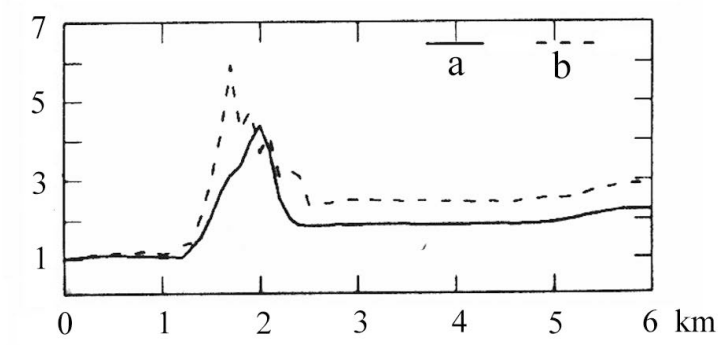

(c)

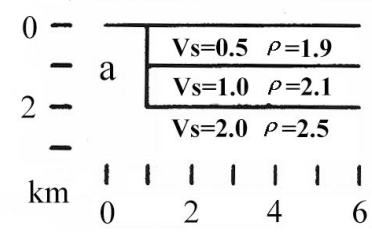

(a)

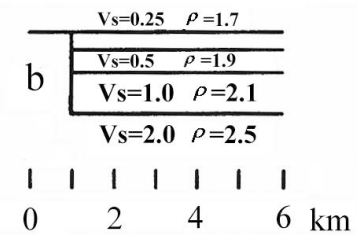

(b)

Figure 5. Simulations for propagation wave field and parameters of basin structure under the plane wave incidence case from the bottom. Simulated soil amplification ratios (c) for different half basin structures with 3 layers (a) and 4 layers (b).

showed that the physical interference between the body wave and the secondary surface wave significantly amplified the ground motions and caused in the peck value acceleration besides the geometrical focusing due to the basin corner. The sites of peak accelerations due to the geometrical focusing were attributable to the angles of corners and azimuths of incidence waves. The location of peak ground motion due to the wave interference was attributable to the apparent velocity difference rather than the velocity difference between the secondary surface wave and the body wave. Some peak ground motion occurred away from the source region. This implies that the geometrical structure in the heterogeneous medium and the wave interference more strongly affects the ground motion than the distance function.

The late-arrival waves of multireflection waves can also resulted in the peak ground motion besides the refraction wave from the rock layer. A series of waves with great amplitudes oscillating up-down within the basin shook the buildings time after time, extending the period shaking the buildings, resulting in heavy seismic damage.

The body wave refracted into the basin from the rock layer probably causes another surface wave. The supercritical reflection of the body wave within the surface layer over the half infinite rock layer is taken as the physical mechanism generating the surface wave. The multi-reflection wave propagating in the soil basin structure with velocity $\mathrm{V}_{\mathrm{s} 1}$ (in Table 1 ) over the half infinite rock space with velocity $\mathrm{V}_{\mathrm{s} 2}$ (in Table 1 ) in Figure 2 is similar to the case of the supercritical reflection $\left(\mathrm{V}_{\mathrm{s} 1}<\mathrm{V}_{\mathrm{s} 2}\right)$ of the body wave within the surface layer. It probably is a case of the Love wave propagating in the single layer over the half infinite space indeed.

The spectral analysis of waveform with great wave amplitude is important for the aseismic study and mitigating damage. The spectral analyses of various waveforms during the period with great wave amplitude in Figure 4 indicated that the frequencies of the seismic phases propagating in basin are different from one another. The frequency of the late-arrival waves of multireflection might change during propagation, occurring likely dispersion of the surface wave. The frequency domains of waveforms during the period of great wave amplitude for multi-wave phase get lower and lower according to the sequence of the wave phase arriving. The late-arrival conversion waves with various frequencies widened the frequency band of waves shaking buildings and could seriously damage the buildings with the corresponding intrinsic frequencies.

\section{Acknowledgements}

This study was supported financially by the Natural Science Foundation of China (Research Project No. 41374052). 


\section{References}

[1] Ugurhan, B., Askan, A., Akinci, A. and Malagnini, L. (2012) Strong-Ground-Motion Simulation of the 6 April 2009 L'Aquila, Italy Earthquake. Bulletin of the Seismological Society of America, 102, 1429-1445. http://dx.doi.org/10.1785/0120110060

[2] Liu, S.L., Li, X.F., Wang, W.S., Lu, M.W. and Zang, M.G. (2013) Optimal Symplectic Scheme and Generalized Discrete Convolutional Differentiator for Seismic Wave Modeling. Chinese Journal Geophysics, 56, 2452-2462, http://dx.doi.org/10.6038/cjg20130731

[3] Graves, R.W. (1998) Three-Dimensional Finite Difference Modeling of the San Andreas Fault: Source Parameterization and Ground Motion Levels. Bulletin of the Seismological Society of America, 88, 881-897.

[4] Liu, Y.S., Teng, J.W., Liu, S.L. and Xu, T. (2013) Explicit Finite Element Method with Triangle Meshes Stored by Sparse Format and Its Perfectly Matched Layers Absorbing Boundary Condition. Chinese Journal Geophysics, 56, 3085-3099. http://dx.doi.org/10.6038/cjg20130921

[5] Zhao, Z., Liu, L.B. and Barrowes, B. (2012) Radar Wave Simulation Using Three-Dimensional Multi-Region Pseudospectral Time Domain Algorithm with Directional Kirchhoff Integration Surface, Ground Penetrating Radar (GPR). 14th International Conference on Ground Penetrating Radar (GPR), Shanghai, 4-8 June 2012, 248-253. http://dx.doi.org/10.1109/ICGPR.2012.6254869

[6] Wang, M.X., Yang, D.H. and Song, G.J. (2012) Semi-Analytical Solutions and Numerical Simulations of 2D SH Wave Equation. Chinese Journal of Geophysics, 55, 914-924. (In Chinese)

[7] Yang, B. and Hesthaven, J.S. (2000) Multidomain Pseudospectral Computation of Maxwell’s Equations in 3-D General Curvilinear Coordinates. Applied Numerical mathematics, 33, 281-289. http://dx.doi.org/10.1016/S0168-9274(99)00094-X

[8] Pavlenko, O.V. (2013) Simulation of Ground Motion from Strong Earthquakes of Kamchatka Region (1992-1993) at Rock and Soil Sites. Pure and Applied Geophysics, 170, 571-595. http://dx.doi.org/10.1007/s00024-012-0529-x

[9] Jing, H., Deng, Z.H., Gao, X.L., Chen, M.H. and Yang, Z.Z. (2013) Numerical Modeling of Earthquake Generating Processes in the Japan Subduction Slab. Chinese Journal Geophysics, 56, 2303-2312.

[10] Zhao, Z.X., Zhao, Z., Xu, J.R. and Kubota, R. (2006) Synthetic Seismograms near Earthquake Fault Using Simulated Green’s Function Method. Chinese Science Bulletin, 51, 3018-3025. http://dx.doi.org/10.1007/s11434-006-2190-1

[11] Yadav, R.B.S., Tsapanos, T.M., Yusuf, B. and Koravos, G.C. (2013) Probabilistic Appraisal of Earthquake Hazard Parameters Deduced from a Bayesian Approach in the Northwest Frontier of the Himalayas. Pure and Applied Geophysics, 170, 283-297. http://dx.doi.org/10.1007/s00024-012-0488-2

[12] Irikura, K. (1995) Causative Fault, Strong Ground Motion and Damage from the 1995 Hyogoken Nanbu Earthquake. Butsuri-Tansa, 48, 463-489. (In Japanese with English Abstract)

[13] Zhang, H., Zhou, Y.Z., Wu, Z., Yan, Z.Z., Chen, S., Jing, H.M., Xu, X.W. and Shi, Y.L. (2009) Finite Element Analysis of Seismic Wave Propagation Characteristics in Fuzhou Basin. Chinese Journal of Geophysics, 52, 1270-1279. (In Chinese)

[14] Furumura, T., Kennett, B.L.N. and Takenaka, H. (1998) Parallel 3-D Pseudo-Spectral Simulation of Seismic Wave Propagation. Geophysics, 63, 279-288. http://dx.doi.org/10.1190/1.1444322

[15] Shahar, S.K., Tsesarsky, M., Louie, J.N. and Gvirtzman, Z. (2012) Simulation of Seismic-Wave Propagation through Geometrically Complex Basins: The Dead Sea Basin. Bulletin of the Seismological Society of America, 102, 17291739. http://dx.doi.org/10.1785/0120110254

[16] Yang, R.H., Chang, X. and Liu, Y.K. (2009) Viscoelastic Wave Modeling in Heterogeneous and Isotropic Media. Chinese Journal Geophysics, 52, 2321-2327. (In Chinese)

[17] Zhao, Z.X. and Xu, J.R. (2003) Staggered Grid Real Value FFT Differentiation and Application. Chinese Journal of Geophysics, 46, 234-240. (In Chinese with English Abstract) http://dx.doi.org/10.1002/cjg2.348

[18] Fujiwara, H. and Takenaka, H. (1994) Calculation of Surface Waves for a Thin Basin Structure Using a Direct Boundary Element Method with Normal Modes. Geophysical Journal International, 117, 69-91. http://dx.doi.org/10.1111/j.1365-246X.1994.tb03304.X

[19] Zeng, Y.Q. and Liu, Q.H. (2004) A Multidomain PSTD Method for 3D Elastic wave Equations. Bulletin of the Seismological Society of America, 94, 1002-1015. http://dx.doi.org/10.1785/0120030103

[20] Pitraka, A., Irikura, K. and Iwata, T. (1998) Three-Dimensional Simulation of the Near-Fault Ground Motion for the 1995 Hyogoken Nanbu (Kobe), Japan, Earthquake. Bulletin of the Seismological Society of America, 88, 428-440.

[21] Boatwright, K. (2001) Correlation of Ground Motion and the Intensity of the 17 January 1994 Northridge, California Earthquake. Bulletin of the Seismological Society of America, 91, 739-752. http://dx.doi.org/10.1785/0119990049 
[22] Zhao, Z., Zhao, Z.X. and Xu, J.R. (2010) Interference between Seismic Body Wave and Secondary Surface Wave Resulting in the Peak Collapse Ratios of Buildings. Journal of Applied Geophysics, 72, 1-9. http://dx.doi.org/10.1016/j.jappgeo.2010.06.001

[23] Zhao, Z.X., Xu, J.R. and Yang, W.C. (2004) Simulations of Reflection Seismic Profile of Borehole Area of Chinese Continental Scientific Drilling. Acta Petrologica Sinica, 20, 127-136. (In Chinese with English Abstract)

[24] Rodgers, A.J., Petersson, N.A. and Sjogreen, B. (2010) Simulation of Topographic Effects on Seismic Waves from Shallow Explosions near the North Korean Nuclear Test Site with Emphasis on Shear Wave Generation. Journal of Geophysical Research, 115. http://dx.doi.org/10.1029/2010JB007707

[25] Zhao, Z.X., Xu, J.R. and Horiuchi, H. (2001) Differentiation Operation in the Wave Equation for the Pseudospectral Method with a Staggered Grid. Earth Planets Space, 53, 327-332. http://dx.doi.org/10.1186/BF03352389

[26] Kosloff, D., Reshef, M. and Loewenthal, D. (1984) Elastic Wave Calculations by the Fourier Method. Bulletin of the Seismological Society of America, 74, 875-891.

[27] Wang, Y., Takenaka1, H. and Furumura, T. (2001) Modeling Seismic Wave Propagation in a Two-Dimensional Cylindrical Whole-Earth Model Using the Pseudospectral Method. Geophysical Journal International, 145, 689-708. http://dx.doi.org/10.1046/j.1365-246x.2001.01413.x

[28] Reshef, M., Kosloff, D., Edwards, M. and Hsiung, C. (1988) Three-Dimensional Elastic Modeling by the Fourier Method. Geophysics, 53, 1184-1193. http://dx.doi.org/10.1190/1.1442558

[29] Somerville, P.G., Smith, N.F. and Graves, R.W. (1997) Modification of Empirical Strong Ground Motion Attenuation Relations to Include the Amplitude and Duration Effects of Rupture Directivity. Seismological Research Letters, 68, 199-222. http://dx.doi.org/10.1785/gssrl.68.1.199

[30] Cerjan, C., Kosloff, D., Koskoff, R. and Reshef, M. (1985) A Nonreflecting Boundary Condition for Discrete Acoustic and Elastic Wave Equations. Geophysics, 50, 705-708. http://dx.doi.org/10.1190/1.1441945

[31] Zhao, Z.X., Kubota, R., Suzuki, F. and Iizuka, S. (1997) Crustal Structure in the Southern Kanto-Tokai Region Derived from the Tomographic Method for Seismic Explosion Survey. Journal of Physics of the Earth, 45, 433-453. http://dx.doi.org/10.4294/jpe1952.45.433

[32] Adcroft, A., Hallberg, R., Dunne, J.P., Samuels, B.L., Galt, J.A., Barker, C.H. and Payton, D. (2010) Simulations of Underwater Plumes of Dissolved Oil in the Gulf of Mexico. Geophysical Research Letters, 37. http://dx.doi.org/10.1029/2010gl044689

[33] Abrahamson, N.A. and Silva, W.J. (1997) Empirical Response Spectral Attenuation Relations for Shallow Crustal Earthquakes. Seismological Research Letters, 68, 94-127. http://dx.doi.org/10.1785/gssrl.68.1.94

[34] Gülerce, Z. and Abrahamson, N.A. (2010) Vector-Valued Probabilistic Seismic Hazard Assessment for the Effects of Vertical Ground Motions on the Seismic Response of Highway Bridges. Earthquake Spectra, 26, 1-18. http://dx.doi.org/10.1193/1.3464548

[35] Saito, T., Ito, Y., Inazu, D. and Hino, R. (2011) Tsunami Source of the 2011 Tohoku-Oki Earthquake, Japan: Inversion Analysis Based on Dispersive Tsunami Simulations. Geophysical Research Letters, 38. http://dx.doi.org/10.1029/2011GL049089

[36] Aki, K. and Richatds, P.G. (1980) Quantitative Seismology, Theory and Methods. Freeman and Company, San Francisco, 331-333.

[37] Zhao, Z.X., Xu, J.R. and Kubota, R. (2004) Effects for Soil Amplification Ratios and Seismic Wave Multiple Interferences for Ground Motion Due to Earthquake. Chinese Science Bulletin, 49, 2405-2414.

http://dx.doi.org/10.1007/BF03183430 